\title{
Crimean-Congo hemorrhagic fever
}

INSERM

\section{Source}

INSERM. (1999). Orphanet: an online rare disease and orphan drug data base. CrimeanCongo hemorrhagic fever. ORPHA:99827

Crimean-Congo hemorrhagic fever (CCHF) is a tick-borne zoonotic disease caused by CCHF virus and characterized by initial fever, headache, and malaise followed by gastrointestinal symptoms and, in severe cases, bleeding, shock, and multi-organ system failure. 